\title{
Chaos in Nonlinear Dynamic Systems: Helicopter Vibration Mechanisms
}

\author{
James H. Taylor ${ }^{1}$, Member, Saied S. Sharif ${ }^{2}$, Member \\ Department of Electrical and Computer Engineering, \\ University of New Brunswick \\ Fredericton, New Brunswick, CANADA E3B 5A3 \\ E-mail: 1) jtaylor@unb.ca, 2) salamat@ee.unb.ca
}

\begin{abstract}
The nonlinear dynamic behavior of a helicopter is considered in this paper, using only real-time flight data analysis. The main objective of this study is to characterize the vibration mechanism(s), based on the analysis of the time-series data of the dynamical system, specifically acceleration for two different airspeeds with a sampling rate of $1024 \mathrm{~Hz}$.

We explore the possibility of the presence of chaotic behavior in the time-series data, using a systematic, detailed approach. Some background in the theory of chaos in nonlinear dynamical systems is discussed, and techniques for the identification of chaos in time-series data are presented. Several topics including delay-coordinate embedding theory, delay time and dimension calculation, and Lyapunov exponent computation for chaotic systems are described. In each section, time-series data sets from the helicopter are analyzed and examined; in some sections classical examples such as the Hénon Map and Lorenz System are also considered to provide illustrative results. Finally, implications regarding the possibility of chaotic behavior in the dynamical system is discussed, and the next steps in this study are presented.
\end{abstract}

\section{INTRODUCTION}

An understanding of the identification and control of chaotic systems has improved tremendously in the last decade [1]-[12]. Chaotic behavior occurs in systems with nonlinear dynamics. The recognition of chaos in a complex dynamical system is very complicated. An important characteristic of a chaotic system is its sensitive dependence on initial conditions.

Chaos may exist in classical systems (represented by mathematical models) or real-life systems. Examples of classical systems include the logistic, tent and Hénon maps in the realm of discrete systems, and the Lorenz and Rössler differential equations for continuous-time dynamical systems [2]. Some real-life systems in which chaos has been identified are NMR laser data, human breath rate [3], among others. If a system shows some signs of chaotic behavior, then it may be controlled with some of the methods which are proposed in the literature [3], [4], [5].

In most real-life systems, system behavior is characterized by time-series data available from measurement. The first step in the analysis of time-series data was introduced in Geometry from a time series [6], in which state space reconstruction of time-series data was proposed for the first time.
In delay-coordinate reconstruction, the selection of time delay and dimension are the most important issues [7], [8]. By appropriate selection of time delay and embedding dimension, the time-series data can be reconstructed in the delay-coordinate state space. In this space, the chaotic behavior of the nonlinear dynamical system can be studied. A common method for the identification of chaos in state space systems is to calculate the Lyapunov exponent [4]. In this paper, the time series of a helicopter flight data is analyzed to investigate the possibility of chaotic behavior.

The organization of this paper is as follows. In Section II, the delay-coordinate state space reconstruction of time-series data is discussed. In Section III, the calculation of the optimal time delay for delay-coordinate reconstruction is described and carried out. In Section IV, the dimension calculation of the reconstructed state space is addressed and also executed. In Section V, the computation of Lyapunov exponent for time-series data, and identification of chaos is considered and results presented. In Section VI, the concluding remarks about the helicopter data and the presence of chaos are discussed.

\section{State Space Reconstruction Via DELAY-COORDINATE EMBEDDING}

In most cases, observations of a system are in the form of time-series data, not a state space representation. However, the most effective approaches for studying deterministic dynamical systems involve describing the system in an appropriate state space. For this reason, time-series data should be converted into state space vectors. This procedure is known as state space reconstruction, which is based on a theorem attributed to Takens [9].

For many physical systems, a state space flow model, is not available; rather, observations of the system variables are taken, including measurements of one or several quantities which depends on the current state of the system. Given a scalar signal, $s(t)$, regularly sampled at time interval $\tau_{s}$ starting at some time $t_{0}$, the $n$th sample can be represented as:

$$
s_{n}=s\left(t_{0}+(n-1) \tau_{s}\right)+\eta_{n}, \quad n=1,2, \ldots
$$

where $\eta_{n}$ is the measurement noise. A delay-coordinate reconstruction can be formed by plotting the time series versus a time-delayed version of it. For a 2-dimensional reconstruction, we plot the delay vector $\mathbf{y}_{n}=\left(s_{n}, s_{n-V}\right)$, where $V$ is the lag or sampling delay, i.e., the difference between the adjacent components of the delay vector in 
number of samples. For a $d$-dimensional reconstruction, the delay vector, $\mathbf{y}_{n}$ can be presented as:

$$
\mathbf{y}_{n}=\left[s_{n}, s_{n-V}, \cdots, s_{n-(d-2) V}, s_{n-(d-1) V}\right] .
$$

If the dimension of the reconstructed space, $d$, is larger than twice the box counting dimension, $m$, of the attractor (or the number of active degrees of freedom), the equivalence of the spaces is guaranteed [9]. In some applications, a smaller value of $d$ can also be sufficient [1].

\section{A. Helicopter Flight Time Series Data}

The dynamical system which is considered in this paper is characterized by helicopter test flight data. Two sets of data are examined, each comprised of acceleration signals (calibration withheld) for two different airspeeds (also withheld). The data are sampled when the active vibration control system is switched off.

The time evolution of the first 1000 samples is shown in Figure 1. As a preliminary investigation, the power spectrum of these data was also calculated; the results are presented in Figure 2. As shown in this figure, the highest amplitude pertains to the blade passing frequency; however, we observe that substantial power is also present over a broad range of frequencies. The general sources of broad band frequency content can be random noise or chaos; since the time-series plots appear to be quite clean (Figure 1), the presence of chaos in the time-series data may be suspected.

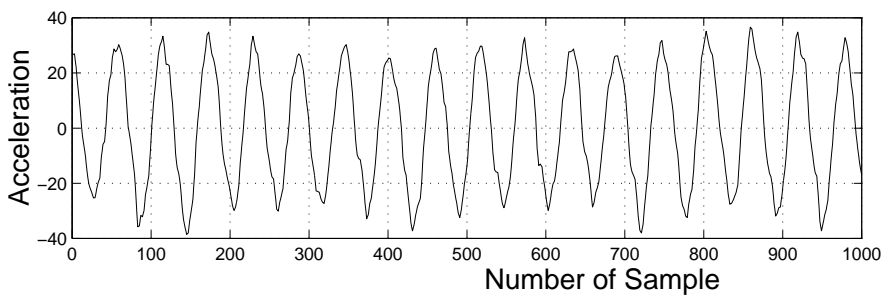

Fig. 1. The time evolution of the first 1000 samples of flight data

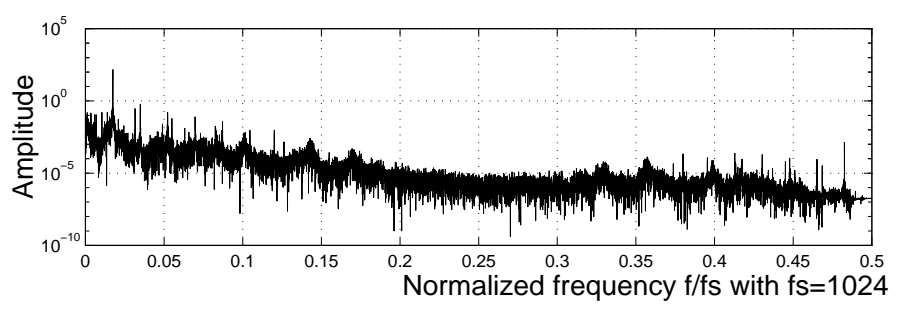

Fig. 2. Power spectrum of flight data

\section{B. Hénon Map and Lorenz System}

For the verification of the obtained results for the flight data, two classical models are also addressed in this report. Hénon Map and Lorenz System, respectively as a discrete and continuous chaotic systems are selected. The Hénon Map can be presented as:

$$
\left\{\begin{array}{l}
x_{n+1}=1-a x_{n}^{2}+y_{n} \\
y_{n+1}=b x_{n}
\end{array}\right.
$$

where $a$ and $b$ are equal to 1.4 and 0.3 , respectively. The Lorenz System can be formulated as:

$$
\left\{\begin{array}{l}
\dot{x}=\sigma(y-x) \\
\dot{y}=x(R-z)-y \\
\dot{z}=x y-b z,
\end{array}\right.
$$

where $\sigma=16.0, R=45.92$, and $b=4.0$. The three state variables of this system are two componenets of temperature and one component of velocity in the convection problem. For comparison of Lorenz System and the flight timeseries data, the time evolution and power spectrum of the three states of this system are shown in Figure 3 and 4, respectively.

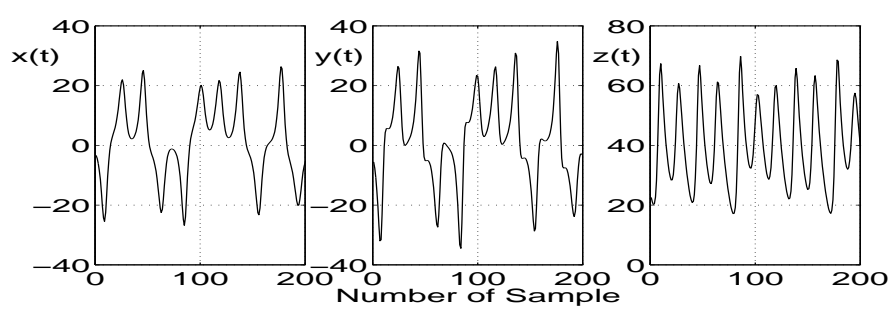

Fig. 3. The time evolution of the three states of Lorenz System

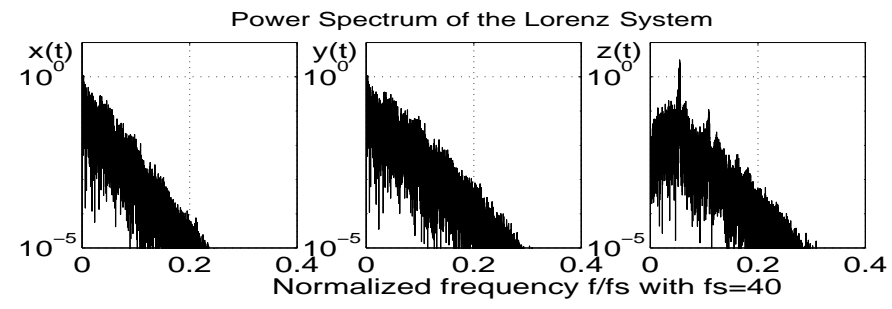

Fig. 4. Power spectrum of the three states of Lorenz System

\section{Calculation of Optimal Time Delay}

From a mathematical point of view, if an infinite amount of infinitely accurate data is available, there is no limit on the choice of sampling delay, except certain multiples of the precise period of a periodic signal. These conditions cannot be met in real-life data, and an optimal choice of sampling delay plays an important role in the analysis of the reconstructed system. An optimal sampling delay should be large enough to give rather independent values for $s_{n}$ and $s_{n-V}$, and not too large that it gives completely independent $s_{n}$ and $s_{n-V}$. Two methods for the optimal selection of sampling delay which give satisfactory results in most applications are discussed below.

\section{A. Time Delay Calculation: Autocorrelation Method}

In this approach, the autocorrelation function of the signal is used to identify the optimal value of sampling delay [10]. A commonly used rule of thumb [5] for the calculation of sampling lag is to set $V$ equal to the sampling lag required for the autocorrelation function to become negative. The problem with this approach is that it is only based on linear statistics, and it does not account for any nonlinear dynamical correlation. 
The autocorrelation function of flight data is shown in Figure 5. It should be noted that this function is periodic, and only part of the first period is shown. The optimal value of sampling delay based on this figure is between $V=14$ and $V=15$.

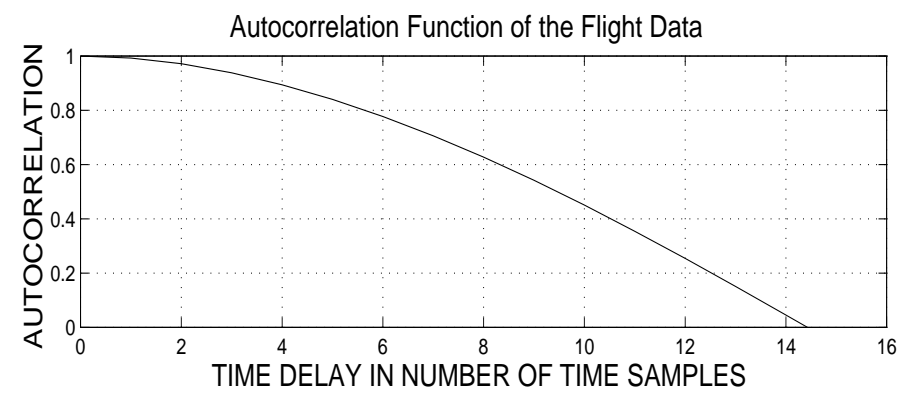

Fig. 5. The autocorrelation function of flight data

\section{B. Time Delay Calculation: Mutual Information Method}

The other method for the calculation of sampling delay is the using of mutual information function [7]. The original concept of mutual information is based on Shannon's information theory, which gives a measure of the general independence of two variables. In other words, this function provides the information about the signal at $s_{n+V}$ given that we know $s_{n}$. The sampling lag related to the first minimum of mutual information function specifies the point where the information about $s_{n+V}$ from knowledge of $s_{n}$ is maximal, or where the redundancy is least. In general, the sampling lag value based on the autocorrelation function is not the same as the value from the mutual information function. In such cases, it is better to select an optimal $V$ inside that interval. Optimal values of $V$ can then be verified through the visualization of the data in a two-dimensional embedding.

The mutual information of $z(t)$ variable from Lorenz System is shown in Figure 6. The first minimum of this function is around $V=4$. This function is almost flat for higher values of $V$. The projection of the time-series data of Lorenz System in a 2-dimensional embedding space also verfies the optimal sampling delay of $V=4$.

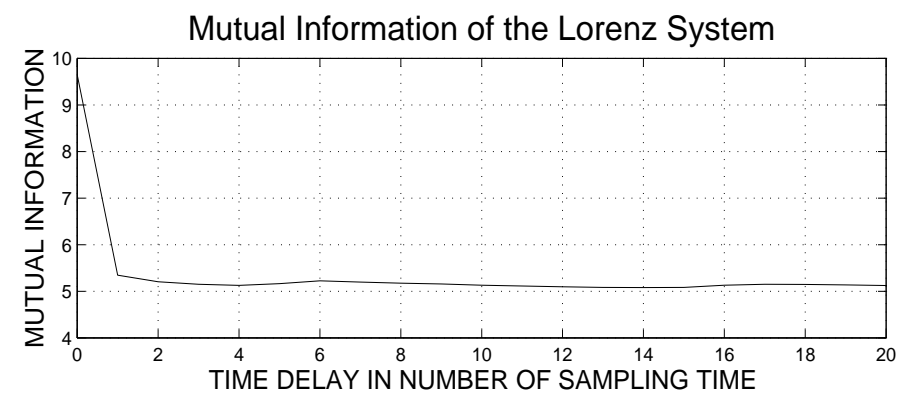

Fig. 6. The mutual information function of $z(t)$ in the Lorenz System

The mutual information of flight data is illustrated in Figure 7. This function has a flat minimum around $V=14$ to $V=17$. Any value in this range should be a good choice

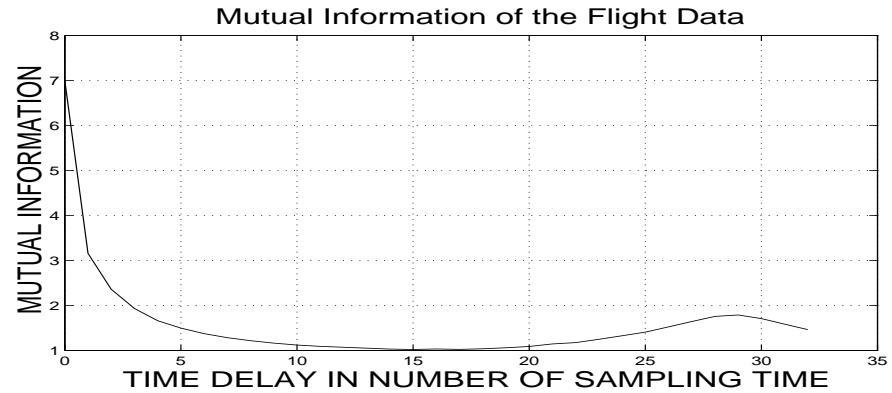

Fig. 7. The mutual information function of flight data

for the sampling delay. From the visualization of data in twodimensional delay coordinates, the optimal value of $V=14$ to 16 can be verified.

\section{Reconstructed State Space Embedding DIMENSION}

The embedding dimension, $d$, is the lowest integer dimension which unfolds the attractor in the projected space with no overlaps. Based on the embedding theorem [9], if the dimension of the attractor defined by the orbits in the original space is $m$, then the attractor can be unfolded in an integer dimensional space of dimension $d$ where $d>2 m$. The false nearest neighbors approach [8] which is one of the most common technique for dimension calculation is used in this paper. The delay-coordinate dimension of the flight timeseries data is calculated with this approach. The result of this calculation is presented in Figure 8. As shown in this figure, the value of false nearest neighbors is very close to zero for $d=6$. This value will be selected for delay-coordinate state space reconstruction of flight data. The delay-coordinate

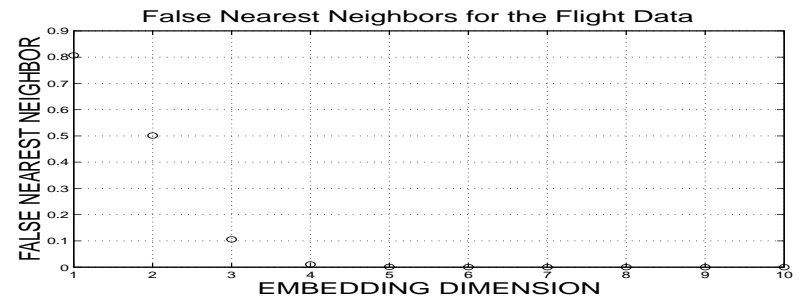

Fig. 8. Calculation of embedding dimension of flight data by false nearest neighbors approach

dimension of Lorenz System is also calculated by using the false nearest neighbors approach. Based on these results, a value of $d=3$ can unfold the Lorenz System. This value is equal to the actual dimension of system, $m=3$. In the following analysis, the value of $d=3$ is used as the mimimum embedding dimension of Lorenz System.

\section{LYAPUNOV EXPONENTS}

In general, the trajectory of a dynamical system starting at an arbitrary initial point can end up at a stable fixed point (sink), a stable closed trajectory (periodic sink), a chaotic orbit, or it may become unstable [2]. A chaotic orbit can be defined as continuously unstable behavior of the system. At 
any point of such an orbit, there are points arbitrary near, that will move away from each other during further iterations. This behavior is in marked contrast to that of a stable limit cycle, where nearby points converge. To discriminate between these phenomena (stable limit cycles and chaotic orbits), the Lyapunov number (or Lyapunov exponent) can give us a useful measure of the convergence or divergence (chaotic behavior) of the system's orbits. The Lyapunov number is defined as the average per-step divergence rate of nearby points along a system's orbits, and the Lyapunov exponent is the natural logarithm of the Lyapunov number.

In a one-dimensional map, a single Lyapunov number gives a measure of separation rates of nearby points along the real line. For maps on $\Re^{m}$ for $m>1$, however, nearby points may diverge in one direction and converge in another. Therefore, in an $m$-dimensional map, each orbit has $m$ Lyapunov numbers. These numbers measure the rate of expansion/contraction from the current point along $m$ orthogonal directions. In general, the maximal Lyapunov number/exponent is the most important one for identifying chaotic behavior of a system.

The Lyapunov exponent is the average of exponential expansion/contraction rates over the whole time-series data. This value can be measured as the average rate of the expansion/contraction, $D_{l}$, versus time steps, $\Delta k$. In this calculation, only the points in the $\epsilon$-neighborhood of each initial point are considered [11].

\section{A. Calculation of the Maximal Lyapunov Exponent for Hénon Map and Lorenz System}

For verification of our analysis in the computation of maximal Lyapunov exponent, $\lambda_{1}$, two classical chaotic examples are examined. As an example of a discrete system, the value of $\lambda_{1}$ for Hénon Map by the approach of [11] is calculated. The plots of $D_{l}$ for this map for $d=2,3,4$, and several values of $\epsilon$ is shown in Figure 9. The reported value of $\lambda_{1}$ in [12] is equal to 0.418. A dashed line with this slope is shown in Figure 9. As can be seen, the slope of $D_{l}$ for different values of $d$ and $\epsilon$ is very close to the slope of the dashed line.

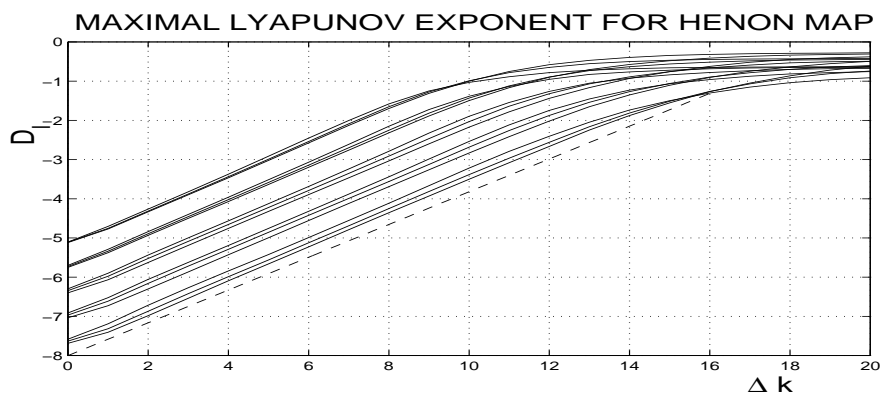

Fig. 9. Maximal Lyapunov exponent calculation of Hénon Map by the approach of [11]

The maximal Lyapunov Exponent of $x(t)$ and $z(t)$ of Lorenz System is also computed by using the approach of [11]. The value of $D_{l}$ is calculated for $d=3,4,5,6$, and several values of $\epsilon$. Due to space limitation, only the plots for $z(t)$ are illustrated in Figure 10. The slope of $D_{l}$ for $x(t)$ is in accordance with the reported value in the literature, $\lambda_{1}=1.5$, [12]. The dashed line in plots of Figure 10 has a slope equal to $\lambda_{1}=1.14$. The fluctuation of $D_{l}$ in these plots are related to the values of $d>3$ and small values of $\epsilon$.
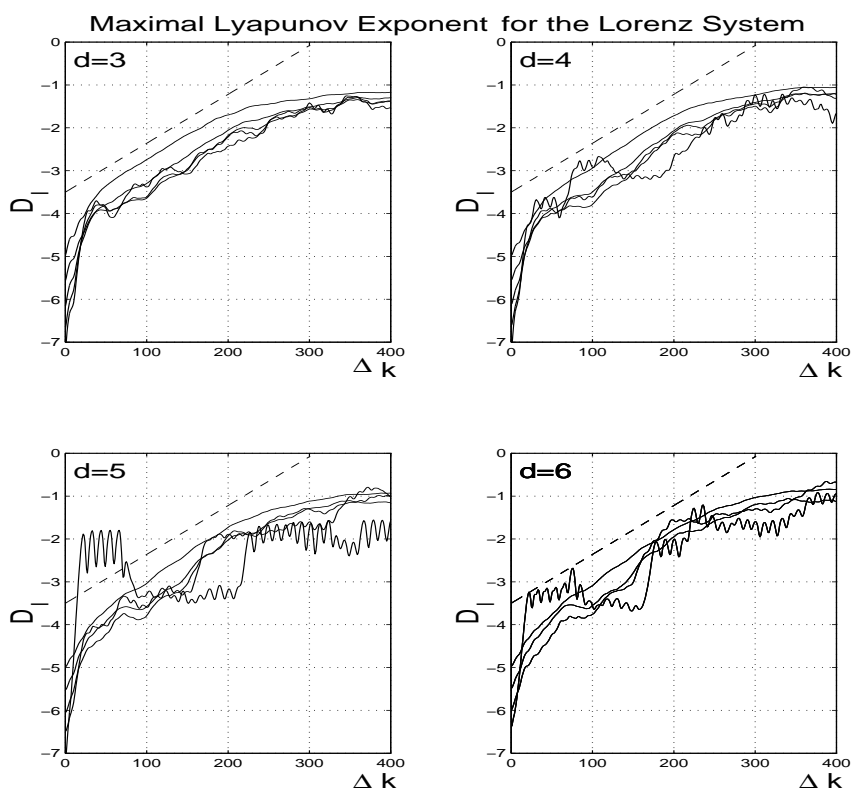

Fig. 10. Maximal Lyapunov exponent calculation of $z(t)$ in the Lorenz System by the approach of [11]

\section{B. Calculation of the Maximal Lyapunov Exponent for Flight Data}

The flight data set is also analyzed by using the method explained in [11]. The sampling delay of $V=14$, and the minimum dimension of $d=6$ are selected. The value of $D_{l}(\Delta k)$ is calculated for different values of $d$ and $\epsilon$.

In the approach followed from [11], the value of $D_{l}(\Delta k)$ is calculated for neighborhood sizes of $\epsilon=6$ and $\epsilon=8$, and five different dimensions $d=6,7, \cdots, 10$, resulting in 10 cases. All the plots are presented in Figure 11.

In this figure, we note that a similar behavior is obtained in each case. The initial strong fluctuation of $D_{l}$ are due to the presence of quasiperiodicity in the dynamical system. Underlying these fluctuations, a distinct linear increase is apparent, as shown by the dashed line. The slope of this line gives the estimated value of the maximal Lyapunov exponent. The average calculated value of maximal Lyapunov exponent from Figure 11 is $\lambda_{1}=5.7 * 10^{-3} 1 /$ time step or $\lambda_{1}=5.84$ $1 /$ second. It should be noted that in real-life time series, the number of data points and sampling time can also have some effects on the computed Lyapunov exponent. For the consideration of this case, a new data set by removing every other point of the original data is created. By using the same approaches, the new optimal sampling delay, $V$, and embedding dimension, $d$, are estimated. The plots for the new data set for four different values of dimension, 


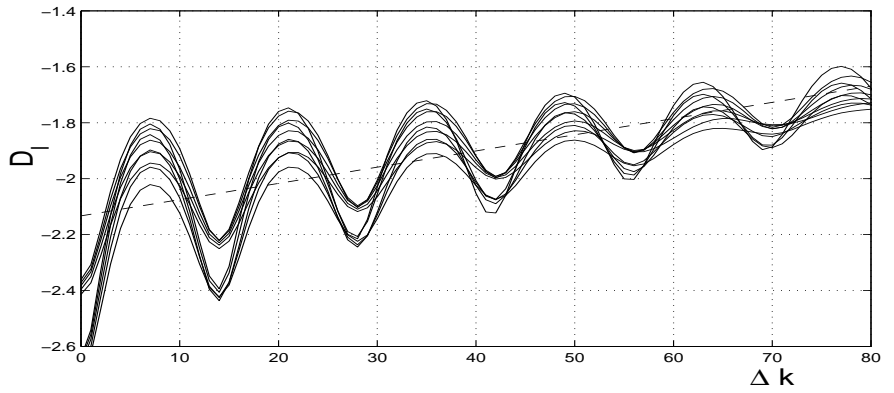

Fig. 11. Maximal Lyapunov exponent calculation for the flight data by the method of [11]

$d=6,7, \cdots, 10$ are presented in Figure 12. The dashed line has the slope of $\lambda_{1}=9.6 E-31 /$ time step or 4.92 $1 /$ second. From this figure, it can be concluded that the sampling time can have some small effects on the calculated value of $\lambda_{1}$.

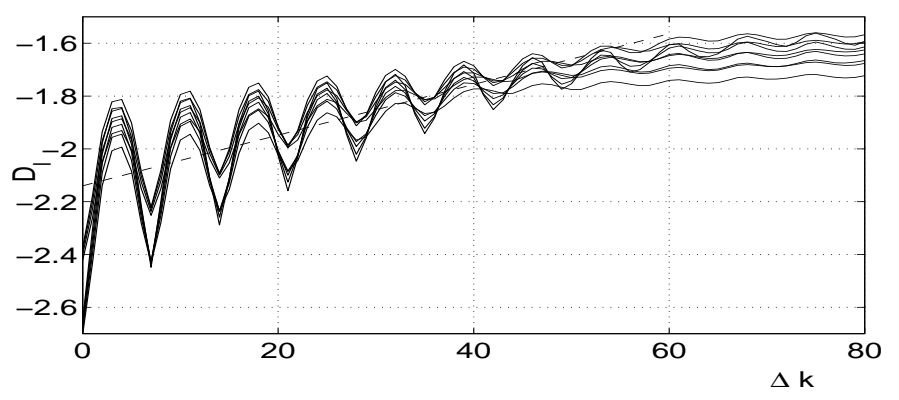

Fig. 12. Maximal Lyapunov exponent computation for half of the flight data

\section{CONCLUSION}

A complete analysis of a helicopter flight data has been performed in order to investigate the possibility of chaotic behavior. The motivation of this study is to find the vibrational characteristics of a helicopter during flight, and to determine the best way to reduce these vibrations. Two sets of flight data related to the acceleration of helicopter for different airspeeds were considered. The data were sampled at $f_{s}=1024 \mathrm{~Hz}$ at the nominal rotor speed of $f_{n}=3.57 \mathrm{~Hz}$, when the active vibration control of helicopter is switched off.

As a first step, the time-series data was inspected in the frequency domain. The blade passing frequency of $5 f_{n}=17.85$ $\mathrm{Hz}$ has the highest amplitude in the power spectrum of the data; however, significant power over a broad range of frequencies is also present. The general sources of broad band frequency content can be random noise or chaos. For this reason, the presence of chaos in the time-series data may be suspected.
One of the major characteristics of chaotic systems is their sensitive dependence of their trajectory to the initial conditions. Two trajectories with very close initial conditions can move apart completely. This characteristic can be quantified by the Lyapunov exponent; in particular, chaotic systems have a maximal Lyapunov exponent greater than zero. In order to calculate the Lyapunov exponent, the given timeseries data were used to reconstruct a state space representation. The delay-coordinate embedding approach was used for this purpose. The selection of sampling delay and dimension of the embedding space were the main considerations; these are thoroughly discussed.

The optimal sampling delay for the flight time-series data was calculated using the autocorrelation and mutual information functions. The final selection was verified by inspecting the data embedded in a 2-dimensional delaycoordinate state space. The dimension of the flight data was computed by the false nearest neighbors approach. These parts of the analysis are well understood and appear to have produced significant new understanding and results.

The Lyapunov exponent of the embedded system was calculated by the methods proposed in the literature and using software supplied by the authors. The maximum Lyapunov exponent derived from the flight data was equal to $\lambda_{1}=$ $5.7 * 10^{-3} 1 /$ time step or $\lambda_{1}=5.841 /$ second. This value is greater than zero, and shows the exponential divergence of nearby points. As a result, the system appears to have a chaotic behavior. This work is still considered speculative, and further study, including methods of noise reduction, is planned.

\section{REFERENCES}

[1] H. D. I. Abarbanel, Analysis of Observed Chaotic Data, Springer, 1996.

[2] K. T. Alligood, T. D. Sauer, J. A. Yorke, An Introduction to Dynamical Systems, New York: Springer-Verlag, 1997.

[3] Nonlinear time series analysis, by Holger Kantz, Maz Planck Institute for Physics of Complex Systems, Dresden Thomas Schreiber, Physics Department, University of Wuppertal, 1997.

[4] E. Ott, Chaos in Dynamical Systems, Cambridge University Press, 1993.

[5] E. Ott, T. Sauer, J. A. Yorke, Coping with Chaos: Analysis of Chaotic Data and the Exploitation of Chaotic systems, New York, Wiley, 1994.

[6] N. Packard, J. Crutchfield, J. D. Farmer, R. Shaw, "Geometry from a time series", American Physical Society, 1980, pp. 712-715.

[7] A. M. Fraser, H. L. Swinney, "Independent coordinates for strange attractors from mutual information", Physical Review A, 33, 1986, pp. 1134.

[8] M. B. Kennel, R. Brown, and H. D. I. Abarbanel, "Determining embedding dimension for phase-space reconstruction using a geometrical construction", Physical Review A, 45, 1992, pp. 3403.

[9] F. Takens, "Detecting strange attractors in turbulence", in Dynamical Systems and Turbulence, Edited by D. A. Rand and L.-S. Young, Warwick 1980.

[10] Casdagli, M., "Chaos and deterministic versus stochastic nonlinear modeling" Journal of the Royal Statistical Society B, 54, 1991, pp. 303

[11] H. Kantz "A robust method to estimate the maximal Lyapunov exponent of a time series", Physical Review A, 185, 1994, pp. 77.

[12] M. T. Rosenstein, J. J. Collins, C. J. De Luca, "A practical method for calculating largest Lyapunov exponents from small data sets", Physica $D, 65,1993$, pp. 117. 